\title{
SEMIORDERINGS AND STABILITY INDEX UNDER FIELD EXTENSIONS
}

\author{
KARIM JOHANNES BECHER, DAVID B. LEEP, AND CLAUS SCHUBERT
}

\begin{abstract}
We study the behavior of orderings, semiorderings, and the stability index under field extensions.
\end{abstract}

Classification (MSC 2000): 11E10, 12F05, 12F20, 12J15

\section{INTRODUCTION}

In this article we want to compare different properties of an extension of real fields, in terms of extendibility of orderings, semiorderings, and the behavior of the stability index.

Semiorderings were introduced by Prestel [11] as a means to obtain a localglobal principle for weak isotropy of quadratic forms over a real field. Around the same time Bröcker [4] introduced a field invariant, referred to in the literature as the (reduced) stability index. While defined in terms of quadratic forms, the stability index is characterized in $[4,(3.19)]$ in terms of general valuations and the corresponding henselizations. In $[4,(4.8)]$ an upper bound for the stability index of an extension field in terms of the transcendence degree and the stability index of the ground field is given. Fans were introduced by Becker and Köpping [2] and used in [5] for a different approach to the stability index. See also [9] for a treatment of these concepts.

One main aim of this article is to decide for certain types of real field extensions whether the stability index may decrease, with special attention to algebraic extensions.

In Section 2 we study fans in relation to semiorderings, with an application in (2.8) to enumerating the number of extensions of a semiordering to a quadratic field extension in a particular case, showing that there is no upper bound on the number of extensions. The problem of determining when fans can be extended to a field extension deserves further study.

In Section 3 we will compare two conditions for a field extension $L / K$. If every ordering of $K$ extends to an ordering of $L$ we say that $L / K$ preserves orderings. If every semiordering of $K$ extends to a semiordering of $L$ we say that $L / K$ is totally positive. As we shall see in (3.4), any totally positive extension preserves orderings. We will show in (3.9) that both properties are equivalent for a Galois

Date: April 17, 2012. 
extension. In general, a field extension that preserves orderings is not necessarily totally positive. We give examples of finite extensions that show the difference between these two properties, answering a question raised in $[1,(3.7)]$. In (3.10) we obtain an easy example of such an extension of degree 16. In (3.12) we describe a class of quartic extensions that preserve orderings, and we show in (3.14) that not every extension in this class is totally positive.

In Section 4 we study the stability index of a field, defined as the supremum on the degrees of its fans, and we study the behavior of this field invariant under field extensions that preserve orderings. We show that the stability index cannot drop over a totally positive extension (4.2) or over a finite extension that preserves orderings (4.5), but that it may drop for a general field extension that preserves orderings (4.3).

In Section 5 we show that the stability index may drop under a real quadratic extension. We give a simplified and more explicit version of Prestel's construction in [12] for a particular case, allowing us to show in (5.3) that any real field $k$ has a real extension field with almost the same properties, having in addition a quadratic field extension with exactly two orderings and where every sum of squares is equal to a sum of two squares. Based on this we determine in (5.7) all possible pairs of values for the stability indices in a real quadratic field extension. It turns out that there may be an arbitrary decrease except to zero in a real quadratic extension. This answers a question raised in [4, p. 251].

\section{FANS}

Let $K$ always denote a field of characteristic different from 2 . Let $\sum K^{2}$ denote the subgroup of $K^{\times}$given by the nonzero sums of squares in $K$. The field $K$ is said to be real if $-1 \notin \sum K^{2}$, nonreal otherwise.

We use the notation $\pm S=S \cup-S$ for any subset $S \subseteq K$. If $S \subseteq K$ is multiplicatively closed and $K^{\times 2} \subseteq S$, we write $S^{\times}=S \cap \overline{K^{\times}}$. A preordering of $K$ is a subset $T \subseteq K$ such that $K^{2} \subseteq T, T+T \subseteq T, T T \subseteq T$, and $-1 \notin T$; if in addition $K= \pm T$, then $T$ is called an ordering of $K$. The set of all orderings of $K$ is denoted by $X(K)$. For a preordering $T$ of $K$ we write $T^{\times}=T \backslash\{0\}$, which is a subgroup of $K^{\times}$, and $X_{T}(K)=\{P \in X(K) \mid T \subseteq P\}$. By [10, Chap. VIII, (9.6)] any preordering $T$ of $K$ satisfies $T=\bigcap_{P \in X_{T}(K)} P$. If $K$ is real, then $\sum K^{2} \cup\{0\}$ is the smallest preordering of $K$. (If $K$ is nonreal then $\sum K^{2}=K^{\times}$and $K$ has no preordering.)

A semiordering of $K$ is a subset $S \subseteq K$ such that $1 \in S, S+S \subseteq S, K^{2} S \subseteq S$, $S \cap-S=0$, and $K= \pm S$. Hence, an ordering is a semiordering that is closed under multiplication.

2.1. Proposition. Let $T$ be a preordering of $K$. The following are equivalent:

(i) For any subgroup $H$ in $K^{\times}$of index 2 containing $T^{\times}$and with $-1 \notin H$, the set $H \cup\{0\}$ is an ordering of $K$. 
(ii) For any subgroup $H$ of $K^{\times}$containing $T^{\times}$and with $-1 \notin H$, the set $H \cup\{0\}$ is a preordering of $K$.

(iii) For any $a \in K^{\times} \backslash \pm T$ we have $T+a T=T \cup a T$.

(iv) Any set $M \subseteq K$ with $T \cdot M \subseteq M$ and $M \cap-M=\{0\}$ is additively closed.

(v) Any set $S \subseteq K$ such that $T \cdot S \subseteq S, S \cap-S=\{0\}, K=S \cup-S$ and $1 \in S$ is a semiordering.

Proof: See [2, Satz 20] or [9, (5.1) and (5.5)] for the equivalence of $(i),(i i)$, and (iii). The implications $(i v) \Rightarrow(v) \Rightarrow(i)$ are obvious. To finish the proof we show $($ iii $) \Rightarrow($ iv $)$. Consider a subset $M \subseteq K$ satisfying $T M \subseteq M$ and $M \cap-M=\{0\}$. For $b, c \in M$ we want to show that $b+c \in M$. We can assume that $b c \neq 0$. Set $a=b c$. If $a \in T$, then $b T=c T$ and $b+c \in b(T+T)=b T \subseteq M$. Assume that $a \notin T$. Since $M \cap-M=\{0\}$ we have $a \notin \pm T$. Assuming ( $i i i$ ) we obtain that $T+a T=T \cup a T$, so $b+c \in b(T+a T)=b(T \cup a T)=b T \cup c T \subseteq M$.

A fan of $K$ is a preordering $T$ of $K$ for which the equivalent conditions $(i)-(v)$ in (2.1) hold.

2.2. Corollary. Let $T$ be a fan of $K$ and let $H$ be subgroup of $K^{\times}$containing $T^{\times}$ and with $-1 \notin H$. Then $H \cup\{0\}$ is a fan of $K$.

Proof: $\quad$ Let $T^{\prime}=H \cup\{0\}$. By condition (ii) in (2.1), $T^{\prime}$ is a preordering. Furthermore, it is clear that $T^{\prime}$ inherits property $(i i)$ from $T$.

2.3. Proposition. Let $n \in \mathbb{N}$ and $T$ a preordering of $K$ with $\left[K^{\times}: \pm T^{\times}\right]=2^{n}$. Then $n+1 \leq\left|X_{T}(K)\right| \leq 2^{n}$. Moreover, $T$ is a fan if and only if $\left|X_{T}(K)\right|=2^{n}$.

Proof: We have $\left[K^{\times}: T^{\times}\right]=2 \cdot\left[K^{\times}: \pm T^{\times}\right]=2^{n+1}$. By [10, Chap. VIII, (9.6)] we have $T^{\times}=\bigcap_{P \in X_{T}(K)} P^{\times}$. As $\left[K^{\times}: P^{\times}\right]=2$ for any $P \in X(K)$, it follows that $\left|X_{T}(K)\right| \geq n+1$. There are $2^{n}$ subgroups of $K^{\times}$containing $T^{\times}$and not containing -1 . Therefore $\left|X_{T}(K)\right| \leq 2^{n}$ and equality holds if and only if $T$ is a fan, by Condition $(i)$ in $(2.1)$.

For a fan $T$ we put $\operatorname{deg} T=\operatorname{dim}_{\mathbb{F}_{2}}\left(K^{\times} / \pm T^{\times}\right)$and call this the degree of $T$; if $\operatorname{deg} T$ is finite, it is equal to $\log _{2}\left|X_{T}(K)\right|$ by (2.3). Note that orderings are fans of degree 0 . Any preordering $T$ of $K$ with $\left[K^{\times}: T^{\times}\right]=4$ is a fan of degree 1 . Fans of degree 0 or 1 are called trivial.

2.4. Lemma. Let $T$ be a preordering of $K$. Assume that $\left[K^{\times}: \pm T^{\times}\right]=2^{n}$ and fix $a_{1}, \ldots, a_{n} \in K^{\times}$such that $\left(-T^{\times}, a_{1} T^{\times}, \ldots, a_{n} T^{\times}\right)$is an $\mathbb{F}_{2}$-basis of $K^{\times} / T^{\times}$. Let $\mathcal{P}_{n}$ denote the power set of $\{1, \ldots, n\}$. For any function $\sigma: \mathcal{P}_{n} \longrightarrow\{ \pm 1\}$ with $\sigma(\emptyset)=1$, the set

$$
S=\bigcup_{I \in \mathcal{P}_{n}}\left(\sigma(I) \prod_{i \in I} a_{i}\right) T
$$

satisfies $T S \subseteq S, S \cap-S=\{0\}, K=S \cup-S$ and $1 \in S$. Conversely, any subset $S \subseteq K$ with these properties is given by such a function $\sigma$. 
Proof: This is straightforward to verify.

Given a preordering $T$, a semiordering $S$ of $K$ is called a $T$-semiordering if $T S \subseteq S$.

2.5. Corollary. Let $n \in \mathbb{N}$ and let $T$ be a preordering of $K$ with $\left[K^{\times}: \pm T^{\times}\right]=2^{n}$. The number of $T$-semiorderings of $K$ is at most $2^{2^{n}-1}$. Moreover, this number equals $2^{2^{n}-1}$ if and only if $T$ is a fan.

Proof: Let $\mathcal{S}$ denote the set of subsets $S \subseteq K$ such that $T S \subseteq S, S \cap-S=\{0\}$, $K=S \cup-S$ and $1 \in S$. By (2.4) we have $|\mathcal{S}|=2^{2^{n}-1}$. Any $T$-semiordering of $K$ belongs to $\mathcal{S}$. Moreover, by Condition $(v)$ in $(2.1), T$ is a fan if and only if every element of $\mathcal{S}$ is a $T$-semiordering.

2.6. Proposition. Let $L=K((t))$. Any ordering $P$ of $K$ has exactly two extensions to an ordering of $L$, given by $(P \cup t P) L^{2}$ and $(P \cup-t P) L^{2}$. For any preordering $T$ of $K$, the set $T^{\prime}=T L^{2}$ is a preordering of $L$ with $T^{\prime} \cap K=T$ and $\left|X_{T^{\prime}}(L)\right|=2\left|X_{T}(K)\right|$. Moreover, if $T$ is a fan of $K$, then $T^{\prime}=T L^{2}$ is a fan of $L$ with $\operatorname{deg}\left(T^{\prime}\right)=\operatorname{deg}(T)+1$.

Proof: See [10, Chap. VIII, (4.11)] for the statement on the extension of orderings from $K$ to $L$. Consider a preordering $T$ of $K$. We define $T^{\prime}$ to be the intersection of all orderings of $L$ that contain $T$. Since each ordering of $K$ extends to $L$, we have that $T^{\prime} \cap K=T$. Since each ordering of $K$ extends in exactly two ways to $L$, it follows that $T^{\prime}$ is a preordering of $L$ and $\left|X_{T^{\prime}}(L)\right|=2\left|X_{T}(K)\right|$.

We need to show that $T^{\prime}=T L^{2}$. Note that $K L^{2} \cup t K L^{2}=L$. Since no element of $t K L^{2}$ is contained in both orderings of $L$ that extend a given ordering of $K$, we obtain that $T^{\prime} \subseteq K L^{2}$. For $x \in K L^{2} \backslash T L^{2}$, there exists $P \in X_{T}(K)$ such that $x \in-P L^{2}$, and then $x$ is not contained in either of the two orderings of $L$ that extend $P$, showing that $x \notin T^{\prime}$. This shows that $T^{\prime} \subseteq T L^{2}$. As the opposite inclusion is straightforward, we obtain that $T L^{2}=T^{\prime}$.

Assume now that $T$ is a fan of $K$. Consider any subgroup $H^{\prime}$ of $L^{\times}$of index 2 with $T^{\prime \times} \subseteq H^{\prime}$ and $-1 \notin H^{\prime}$. Then $H=H^{\prime} \cap K^{\times}$is a subgroup of $K^{\times}$of index 2 with $T^{\times} \subseteq H$ and $-1 \notin H$. Choose $\varepsilon \in\{ \pm 1\}$ such that $\varepsilon t \in H^{\prime}$. Since $L^{\times}=\left(K^{\times} \cup t K^{\times}\right) L^{\times 2}$ we have that $H^{\prime}=(H \cup \varepsilon t H) L^{\times 2}$. As $T$ is a fan, $P=H \cup\{0\}$ is an ordering of $K$. Hence, $H^{\prime} \cup\{0\}=(H \cup \varepsilon t H) L^{\times 2} \cup\{0\}$ is an ordering of $L$, by the first part of the proof. By Condition $(i)$ in $(2.1)$, this argument shows that $T^{\prime}$ is a fan of $L$. Finally, as $\left|X_{T}^{\prime}(L)\right|=2\left|X_{T}(K)\right|$ we obtain that $\operatorname{deg}\left(T^{\prime}\right)=\operatorname{deg}(T)+1$.

2.7. Corollary. Let $n \in \mathbb{N}$ and $L=K\left(\left(t_{1}\right)\right) \ldots\left(\left(t_{n}\right)\right)$. If $T$ is a fan of $K$, then $T^{\prime}=T L^{2}$ is a fan of $L$ with $\operatorname{deg}\left(T^{\prime}\right)=\operatorname{deg}(T)+n$.

Proof: This follows by induction on $n$ from (2.6). 
2.8. Example. Let $n \in \mathbb{N}$. Consider the field $K=\mathbb{Q}\left(\left(t_{1}\right)\right) \ldots\left(\left(t_{n}\right)\right)$ and its quadratic extension $L=\mathbb{Q}(\sqrt{2})\left(\left(t_{1}\right)\right) \ldots\left(\left(t_{n}\right)\right)=K(\sqrt{2})$. It follows from $(2.7)$ that $T=\sum K^{2} \cup\{0\}$ is a fan of $K$ with $\operatorname{deg}(T)=n$ and that $T^{\prime}=T L^{2}=\sum L^{2} \cup\{0\}$ is a fan of $L$ with $\operatorname{deg}\left(T^{\prime}\right)=n+1$. Note that $t_{1}, \ldots, t_{n}$ are representatives of an $\mathbb{F}_{2}$-basis of $K^{\times} / \pm T^{\times}$, and $\sqrt{2}, t_{1}, \ldots, t_{n}$ are representatives of an $\mathbb{F}_{2}$-basis of $L^{\times} / \pm T^{\prime \times}$. By $(2.5) K$ has $2^{2^{n}-1}$ semiorderings and $L$ has $2^{2^{n+1}-1}$ semiorderings. Moreover, any semiordering of $K$ has exactly $2^{2^{n}}$ extensions to $L$, because any choice of signs for the $2^{n}$ elements $\sqrt{2} \cdot t_{1}^{\varepsilon_{1}} \cdots t_{n}^{\varepsilon_{n}}$ with $\left(\varepsilon_{1}, \ldots, \varepsilon_{n}\right) \in\{0,1\}^{n}$ determines one such extension.

We need the following result in Section 5 .

2.9. Proposition. Let $L / K$ be a field extension and let $T^{\prime}$ be a fan of $L$. Then $T^{\prime} \cap K$ is a fan of $K$.

Proof: Note that $T=T^{\prime} \cap K$ is a preordering of $K$. To prove that $T$ is a fan, consider $a \in K^{\times} \backslash \pm T^{\times}$. Since $T^{\prime}$ is a fan and $a \notin \pm T^{\prime}$, we have $T^{\prime}+a T^{\prime}=T^{\prime} \cup a T^{\prime}$, which implies that $T+a T \subseteq\left(T^{\prime}+a T^{\prime}\right) \cap K=\left(T^{\prime} \cup a T^{\prime}\right) \cap K=T \cup a T$, and therefore $T+a T=T \cup a T$. We finish by applying Condition (iii) of (2.1).

\section{EXTENSIONS OF ORDERINGS AND SEMIORDERINGS}

In this section we consider an arbitrary field extension $L / K$. Following [1, Sect. 3] we say that $L / K$ is totally positive if every semiordering of $K$ extends to a semiordering of $L$. We say that $L / K$ preserves orderings if every ordering of $K$ extends to an ordering of $L$.

We rely on [10] for basic results and terminology in quadratic form theory. We will often say 'form' or 'quadratic form' to mean a regular quadratic form. If $\varphi$ and $\psi$ are forms over $K$ we denote by $\varphi \perp \psi$ their orthogonal sum. Given a form $\varphi$ over $K$ we denote by $D_{K}(\varphi)$ the set of nonzero elements of $K$ represented by $\varphi$, and for $n \in \mathbb{N}$ by $n \times \varphi$ the $n$-fold orthogonal $\operatorname{sum} \varphi \perp \cdots \perp \varphi$. A form $\varphi$ over $K$ is called weakly isotropic if $n \times \varphi$ is isotropic for some $n \geq 1$, otherwise strongly anisotropic.

3.1. Proposition. The extension $L / K$ is totally positive if and only if every strongly anisotropic form over $K$ stays strongly anisotropic over $L$.

Proof: See $[1,(3.1)]$.

3.2. Corollary. If every finitely generated subextension of $L / K$ is totally positive, then $L / K$ is totally positive.

Proof: This is immediate from (3.1).

3.3. Lemma. Any ordering of $K$ that extends to a semiordering of $L$ also extends to an ordering of $L$. 
Proof: Consider an ordering $P$ of $K$. If $P$ extends to a semiordering of $L$, then the additive closure of $P L^{2}$ does not contain -1 and thus is a preordering of $L$. Hence $P L^{2} \subseteq P^{\prime}$ for an ordering $P^{\prime}$ of $L$, by [10, Chap. VIII, (9.4)], and then $P^{\prime} \cap K=P$.

3.4. Proposition. Any totally positive extension preserves orderings.

Proof: This is straightforward from (3.3).

As observed in $[1,(3.6)]$ an extension preserving orderings need not be totally positive. We will now compare the two properties for algebraic extensions.

3.5. Proposition. Assume that $L / K$ is a finite extension and let $P$ be an ordering of $K$. The number of extensions of $P$ to an ordering of $L$ is bounded by $[L: K]$, and the two numbers are congruent modulo 2 . If $L / K$ is a Galois extension and $P$ extends to $L$, then $P$ has $[L: K]$ different extensions to an ordering of $L$.

Proof: Let $R$ denote the real closure of $K$ with respect to $P$. Fix an irreducible polynomial $f \in K[X]$ such that $L$ is $K$-isomorphic to $K[X] /(f)$. The extensions of $P$ to an ordering of $L$ are given by the roots of $f$ in $R$ (cf. [13, (3.12)]). As $[L: K]$ is the number of roots of $f$ in the algebraically closed field $R(\sqrt{-1})$ and as the roots in $R(\sqrt{-1}) \backslash R$ are pairwise conjugate under the nontrivial automorphism of $R(\sqrt{-1}) / R$, the first part of the statement follows.

Assume now that $P$ extends to $L$, so that $f$ has a root in $R$. If $L / K$ is a Galois extension, then it follows that all $K$-embeddings of $L$ in $R(\sqrt{-1})$ lie in $R$, so that $f$ has $[L: K]$ roots in $R$, which shows the second part.

In contrast to the situation for orderings described in (3.5), there is no general bound in terms of $[L: K]$ on the number of extensions to $L$ that a semiordering of $K$ may have, as illustrated by the quadratic extension in (2.8).

3.6. Proposition. Any finite extension of odd degree is totally positive.

Proof: By Springer's Theorem [10, Chap. VII, (2.7)] every strongly anisotropic form stays strongly anisotropic over any finite field extension of odd degree. Hence, the statement follows from (3.1).

Note that one may now either use (3.6) and (3.4) or alternatively (3.5) to conclude that every finite extension of odd degree preserves orderings.

3.7. Proposition. Assume that $L=K(\sqrt{d})$ where $d \in K^{\times}$. Then $L / K$ is totally positive if and only if $L / K$ preserves orderings, if and only if $d \in \sum K^{2}$.

Proof: It is shown in $[1,(3.2)]$ that $L / K$ is totally positive if and only if $d \in \sum K^{2}$. Furthermore, an arbitrary ordering of $K$ extends to an ordering of $L$ if and only if it contains $d$. Hence, $L / K$ preserves orderings if and only if $d \in \sum K^{2}$. 
3.8. Theorem. Assume that $L / K$ is a finite extension such that every ordering of $K$ extends in $[L: K]$ different ways to an ordering of $L$. Then $L / K$ is totally positive.

Proof: For any quadratic extension $K^{\prime \prime} / K^{\prime}$ with $K \subseteq K^{\prime} \subseteq K^{\prime \prime} \subseteq L$ it follows easily from the hypothesis using (3.5) that any ordering of $K^{\prime}$ extends in two ways to an ordering of $K^{\prime \prime}$, and thus by (3.7) $K^{\prime \prime} / K^{\prime}$ is totally positive. The statement follows from this when $L / K$ is a 2-extension.

In the general case, write $L=K(\theta)$ and let $f \in K[X]$ be the minimal polynomial of $\theta$ over $K$. The hypothesis means that $f$ splits over any real closure of $K$. Let $M / K$ be the normal closure of $L / K$. Basic Galois theory shows that there exists an extension of odd degree $K^{\prime} / K$ inside $M / K$ such that $L K^{\prime} / K^{\prime}$ is a 2-extension. Let $L^{\prime}=L K^{\prime}=K^{\prime}(\theta)$. The minimal polynomial of $\theta$ over $K^{\prime}$ divides $f$ and therefore splits over every real closure of $K^{\prime}$. Hence, every ordering of $K^{\prime}$ has $\left[L^{\prime}: K^{\prime}\right]$ extensions to $L^{\prime}$. As $L^{\prime} / K^{\prime}$ is a 2-extension, we conclude that $L^{\prime} / K^{\prime}$ is totally positive. As the extension $K^{\prime} / K$ has odd degree, it is totally positive by (3.6). Therefore $L^{\prime} / K$ is totally positive. As $L \subseteq L^{\prime}$ we conclude that $L / K$ is totally positive.

A finite extension $L / K$ may be totally positive without satisfying the hypothesis of (3.8) that every ordering of $K$ extends to $L$ in $[L: K]$ different ways. For example, the extension $\mathbb{Q}(\sqrt[3]{2}) / \mathbb{Q}$ has degree 3 and therefore is totally positive by $(3.6)$, but $\mathbb{Q}(\sqrt[3]{2})$ is uniquely ordered.

3.9. Theorem. A Galois extension is totally positive if and only if it preserves orderings.

Proof: By (3.4) one implication is clear. For the other implication, by (3.2) we only need to consider finite Galois extensions. For a finite Galois extension $L / K$, by (3.5) an ordering of $K$ has either no extension or exactly $[L: K]$ extensions to an ordering of $L$. Hence, if $L / K$ preserves orderings, it follows by (3.8) that $L / K$ is totally positive.

Note that (3.9) generalizes (3.7). We next give an example of an extension of degree 16 that preserves orderings but is not totally positive.

3.10. Example. Let $\alpha$ and $\beta$ denote the positive square roots of 2 and 3 in $\mathbb{R}$. Let $K=\mathbb{Q}((X))((Y))$ and $L=K(\alpha, \beta, \sqrt{\alpha X}, \sqrt{\beta Y})$. Then each of the four orderings of $K$ extends to an ordering of $L$, so $L / K$ preserves orderings. The quadratic form $\langle 1, X, Y,-X Y\rangle$ is strongly anisotropic over $K$. Since $\mathbb{Q}(\alpha, \beta)$ is a number field, the totally indefinite form $\langle 1, \alpha, \beta,-\alpha \beta\rangle$ over $\mathbb{Q}(\alpha, \beta)$ is weakly isotropic. Over $L$ this form becomes isometric to $\langle 1, X, Y,-X Y\rangle$. Hence, $\langle 1, X, Y,-X Y\rangle$ is weakly isotropic over $L$ and thus $L / K$ is not totally positive by (3.1).

We shall now show that certain quartic extensions preserve orderings while not being totally positive. 
3.11. Proposition. Assume that $L=K(\sqrt{a+b \sqrt{d}})$ with $a, b \in K$ and $d \in$ $K^{\times} \backslash K^{\times 2}$. Let $P$ be an ordering of $K$ such that $d \in P$ and $a^{2}-b^{2} d \in-P$. Then $P$ extends to an ordering of $L$ in exactly two different ways.

Proof: As $d \in P$, there are exactly two different orderings $P_{1}$ and $P_{2}$ of $K(\sqrt{d})$ extending $P$. As $(a+b \sqrt{d})(a-b \sqrt{d})=a^{2}-b^{2} d \in-P$, we may assume that $a+b \sqrt{d} \in P_{1} \cap\left(-P_{2}\right)$ and $a-b \sqrt{d} \in\left(-P_{1}\right) \cap P_{2}$. Then $P_{1}$ has exactly two extensions to $L$ whereas $P_{2}$ does not extend to $L$.

3.12. Corollary. Let $d \in \sum K^{2} \backslash K^{\times 2}$ and $a, b \in K$ with $a^{2}-b^{2} d \in-\sum K^{2}$. Then $K(\sqrt{a+b \sqrt{d}}) / K$ preserves orderings.

Proof: This is clear from (3.11).

3.13. Theorem. Let $k$ be a field and $d \in k^{\times} \backslash k^{\times 2}$ and let $a, b \in k^{\times}$such that $a+b \sqrt{d} \notin \sum k(\sqrt{d})^{2}$. Let $K=k(X)$ and $L=K(\sqrt{a+b \sqrt{d}})$. Then the form

$$
\tau=\left\langle 1,\left(X^{2}-d\right),-X(a X-b d),-X(a X-b d)\left(a^{2}-b^{2} d\right)\left(X^{2}-d\right)\right\rangle
$$

is strongly anisotropic over $K$ and becomes isotropic over $L$. In particular, $L / K$ is not totally positive.

Proof: Over $K(\sqrt{d})$, we have $\langle X+\sqrt{d}, X-\sqrt{d}\rangle=2 X \cdot\left\langle 1, X^{2}-d\right\rangle$ and $\langle(a-b \sqrt{d})(X+\sqrt{d}),(a+b \sqrt{d})(X-\sqrt{d})\rangle=2(a X-b d) \cdot\left\langle 1,\left(a^{2}-b^{2} d\right)\left(X^{2}-d\right)\right\rangle$, so that both forms represent $X-\sqrt{d}$ over $L$. Thus $2 X \cdot \tau$ and hence $\tau$ are isotropic over $L$.

To show that $\tau$ is strongly anisotropic over $K$, we consider the two residue forms of $\tau$ with respect to the irreducible polynomial $X^{2}-d$, which are the forms $\langle 1,-(a \pm b \sqrt{d})\rangle$ over $k(\sqrt{d})$. As $a \pm b \sqrt{d} \notin \sum k(\sqrt{d})^{2}$, these residue forms are strongly anisotropic. Thus $\tau$ is strongly anisotropic over $K$.

3.14. Corollary. Let $k$ be a real field, and let $a, b \in k$ and $d \in \sum k^{2} \backslash k^{\times 2}$ be such that $a^{2}-b^{2} d \in-\sum k^{2}$. Let $K=k(X)$ for a variable $X$ over $k$ and $L=K(\sqrt{a+b \sqrt{d}})$. Then $L / K$ preserves orderings but is not totally positive.

Proof: By (3.12) $L / K$ preserves orderings. As $a^{2}-b^{2} d \notin \sum k^{2}$, we have that $a+b \sqrt{d} \notin \sum k(\sqrt{d})^{2}$, so $L / K$ is not totally positive by (3.13).

By $(3.14)$, for example the extension $\mathbb{Q}(\sqrt[4]{2})(X) / \mathbb{Q}(X)$ preserves orderings but is not totally positive.

\section{THE STABILITY INDEX}

The stability index of $K$ is defined to be

$$
\operatorname{st}(K)=\sup \{\operatorname{deg} T \mid T \text { fan of } K\} \in \mathbb{N} \cup\{\infty\} .
$$


By $[5,(2.11)]$, this equals the reduced stability index introduced in [4]. Note that $s t(K)=0$ if and only if $K$ is either nonreal or uniquely ordered.

For a Pfister form $\pi$ over $K$, let $\pi^{\prime}$ denote its pure part, which is defined as the quadratic form over $K$ (unique up to isometry) satisfying $\pi=\langle 1\rangle \perp \pi^{\prime}$.

4.1. Proposition (Bröcker). Let $n \geq 1$. We have $s t(K) \geq n$ if and only if there exists an $n$-fold Pfister form $\pi$ over $K$ such that $\langle-1\rangle \perp \pi^{\prime}$ is strongly anisotropic.

Proof: Using that by $[5,(2.11)]$ the above definition of the stability index is equivalent to the definition in [4], the statement is contained in $[4,(3.17)]$. We give an alternative, more direct proof.

Assume first that $s t(K) \geq n$. It follows easily from (2.2) that there exists a fan $T$ of $K$ of degree $n$. We choose $a_{1}, \ldots, a_{n} \in K^{\times}$such that $\left(-T^{\times}, a_{1} T^{\times}, \ldots, a_{n} T^{\times}\right)$ is an $\mathbb{F}_{2}$-basis of $K^{\times} / T^{\times}$. Let $\mathcal{P}_{n}$ be the power set of $\{1, \ldots, n\}$. Let $S$ denote the $T$-semiordering determined as in (2.4) by the function $\sigma: \mathcal{P}_{n} \longrightarrow\{ \pm 1\}$ with $\sigma(\emptyset)=1$ and $\sigma(M)=-1$ for any $M \in \mathcal{P}_{n} \backslash\{\emptyset\}$. Consider the $n$-fold Pfister form $\pi=\left\langle 1, a_{1}\right\rangle \otimes \cdots \otimes\left\langle 1, a_{n}\right\rangle$ over $K$. Then the form $\langle-1\rangle \perp \pi^{\prime}$ over $K$ represents only elements in $-S$. In particular $\langle-1\rangle \perp \pi^{\prime}$ is strongly anisotropic.

Assume now that $\operatorname{st}(K)<n$. Consider an $n$-fold Pfister form $\pi$ over $K$. By $[9$, (13.1)] there exists an $(n-1)$-fold Pfister form $\rho$ over $K$ such that $(2 \times \rho) \perp-\pi$ is a torsion form. Since $\langle 1,1\rangle \perp-\pi^{\prime}$ is a subform of $(2 \times \rho) \perp-\pi$ of greater than half the dimension, we conclude that $\langle 1,1\rangle \perp-\pi^{\prime}$ is weakly isotropic, and therefore $\langle-1\rangle \perp \pi^{\prime}$ is weakly isotropic.

4.2. Proposition. Let $L / K$ be a field extension. If $L / K$ is totally positive then $\operatorname{st}(L) \geq \operatorname{st}(K)$.

Proof: Suppose that $\pi$ is a Pfister form over $K$ such that $\langle-1\rangle \perp \pi^{\prime}$ is strongly anisotropic. Assuming that $L / K$ is totally positive, $\langle-1\rangle \perp \pi^{\prime}$ remains strongly anisotropic over $L$ by (3.1). Using this, the statement follows from (4.1).

Recall that $K$ is pythagorean if $\sum K^{2}=K^{\times 2}$.

4.3. Theorem. Assume that $K$ is a real field. There exists an extension $L / K$ that preserves orderings such that $L$ is pythagorean and $s t(L)=1$.

Proof: If $K$ is uniquely ordered then let $L=R((t))$ where $R$ is a real closure of $K$. Assume now that $K$ has more than one ordering and let $K_{0}=K$. For $n \in \mathbb{N}$, let $K_{n+1}$ be the compositum over $K_{n}$ of the function fields $K_{n}(\varphi)$ where $\varphi$ runs over the isometry classes of all totally indefinite anisotropic quadratic forms over $K_{n}$; it follows with [6, (3.5)] that any ordering of $K_{n}$ has an extension to $K_{n+1}$. Let $L=\bigcup_{n \in \mathbb{N}} K_{n}$. Then every ordering of $K$ extends to an ordering of $L$. In particular $L$ has more than one ordering, whence $s t(L) \geq 1$. By the construction of $L$ every totally indefinite quadratic form over $L$ is isotropic. In particular, $\langle-1\rangle \perp \pi^{\prime}$ is isotropic for any 2-fold Pfister form $\pi$ over $L$. Hence, $\operatorname{st}(L) \leq 1$ by (4.1). Moreover, $\langle 1,-s\rangle$ is isotropic for all $s \in \sum L^{2}$, hence $L$ is pythagorean. 
A valuation ring is said to be real if its residue field is real.

4.4. Lemma. Let $L / K$ be a field extension that preserves orderings. Let $\mathcal{O}$ be a real valuation ring of $K$. Then there exists a real valuation ring $\mathcal{O}^{\prime}$ of $L$ such that $\mathcal{O}^{\prime} \cap K=\mathcal{O}$. Moreover, given an ordering $P_{0}$ on the residue field of $\mathcal{O}$, the valuation ring $\mathcal{O}^{\prime}$ of $L$ can be chosen such that $P_{0}$ extends to an ordering of the residue field of $\mathcal{O}^{\prime}$.

Proof: Let $\kappa$ be the residue field of $\mathcal{O}$. By the hypothesis $\kappa$ is real. Let $P_{0}$ be an ordering of $\kappa$. By [7, (2.2.5)], there exists an ordering $P$ of $K$ such that $P_{0}=\left\{\bar{x} \mid x \in P \cap \mathcal{O}^{\times}\right\} \cup\{0\}$ and $\mathcal{O}$ is convex in $K$ with respect to the order relation induced by $P$. By the hypothesis, $P$ extends to an ordering $P^{\prime}$ of $L$. Let $\mathcal{O}^{\prime}$ denote the convex hull of $\mathcal{O}$ in $L$ with respect to the order relation given by $P^{\prime}$. By [7, Sect. 2.2.2] $\mathcal{O}^{\prime}$ is a valuation ring of $L$. Since $\mathcal{O} \subseteq \mathcal{O}^{\prime}$ and $\mathcal{O}$ is convex in $K$ with respect to $P=P^{\prime} \cap K$, we obtain that $\mathcal{O}^{\prime} \cap K=\mathcal{O}$. Let $\kappa^{\prime}$ be the residue field of $\mathcal{O}^{\prime}$. By $[7,(2.2 .4)]$ the set $P_{0}^{\prime}=\left\{\bar{x} \mid x \in P^{\prime} \cap \mathcal{O}^{\prime \times}\right\} \cup\{0\}$ is an ordering of $\kappa^{\prime}$ such that $P_{0}^{\prime} \cap \kappa=P_{0}$.

4.5. Theorem. Let $L / K$ be a finite field extension that preserves orderings. Then $\operatorname{st}(K) \leq s t(L) \leq s t(K)+1$.

Proof: The second inequality is shown in $[4,(4.3)]$. To prove the first inequality, we may suppose that $K$ is real and that $s t(L)<\infty$. It suffices to show that $\operatorname{deg}(T) \leq s t(L)$ for any fan $T$ of $K$. Consider a fan $T$ of $K$. By [5, (2.7)] there exists a real valuation ring $\mathcal{O}$ of $K$ such that $\bar{T}=\left\{\bar{x} \mid x \in \mathcal{O}^{\times} \cap T^{\times}\right\} \cup\{0\}$ is a trivial fan of the residue field $\kappa$. By (4.4) there exists a real valuation ring $\mathcal{O}^{\prime}$ of $L$ such that $\mathcal{O}^{\prime} \cap K=\mathcal{O}$. Let $\kappa^{\prime}$ denote the residue field of $\mathcal{O}^{\prime}$. Let $\Gamma=K^{\times} / \mathcal{O}^{\times}$and $\Gamma^{\prime}=L^{\times} / \mathcal{O}^{\prime \times}$. Note that $\Gamma$ is the natural value group of a valuation on $K$ with valuation $\operatorname{ring} \mathcal{O}$ and, similarly, $\Gamma^{\prime}$ is the natural value group of a valuation on $L$ with valuation ring $\mathcal{O}^{\prime}$. Since $\operatorname{st}(L)<\infty$, we obtain using $[3,(4.2)]$ that $\left|\Gamma^{\prime} / 2 \Gamma^{\prime}\right|=2^{r}$ for some $r \in \mathbb{N}$ with $r \leq \operatorname{st}(L)$. As $L / K$ is a finite extension, the natural image of $\Gamma$ in $\Gamma^{\prime}$ has finite index, so we obtain using $[3,(3.4)]$ that $|\Gamma / 2 \Gamma|=\left|\Gamma^{\prime} / 2 \Gamma^{\prime}\right|=2^{r}$. Using that $\Gamma \simeq K^{\times} / \mathcal{O}^{\times}$and further $\mathcal{O}^{\times} T^{\times} / T^{\times} \simeq \mathcal{O}^{\times} /\left(\mathcal{O}^{\times} \cap T^{\times}\right) \simeq \kappa^{\times} / \bar{T}^{\times}$, we obtain that

$$
\left[K^{\times}: T^{\times}\right]=\left[K^{\times}: \mathcal{O}^{\times} T^{\times}\right] \cdot\left[\mathcal{O}^{\times} T^{\times}: T^{\times}\right] \leq|\Gamma / 2 \Gamma| \cdot\left[\kappa^{\times}: \bar{T}^{\times}\right]
$$

and thus $\operatorname{deg}(T) \leq r+\operatorname{deg}(\bar{T})$. As the extension $\kappa^{\prime} / \kappa$ is finite, if $\kappa^{\prime}$ is uniquely ordered, then so is $\kappa$. Since $\operatorname{deg}(\bar{T}) \leq 1$ it follows that $s t\left(\kappa^{\prime}\right) \geq \operatorname{deg}(\bar{T})$. Using $[3$, (4.2)] we conclude that $\operatorname{deg}(T) \leq r+\operatorname{st}\left(\kappa^{\prime}\right) \leq s t(L)$.

In $[11,(2.7)]$ it is shown that, if $L / K$ is a finite field extension and $L$ is uniquely ordered, then $K$ is uniquely ordered. In other terms, for a finite real extension $L / K$ the stability index 'cannot drop to zero': if $\operatorname{st}(L)=0$ then also $s t(K)=0$. We shall see in (5.7) that for quadratic extensions this gives the only constraint for the decrease of the stability index. 


\section{Stability INDEX OF A QUADRATIC FIELD EXTENSION}

In this section we provide a construction for a quadratic field extension having exactly two orderings where the base field has many orderings and any given stability index. This will allow us to determine in (5.7) the possible pairs of values for the stability indices in a quadratic field extension. The results in this section are inspired by results due to Prestel [12] related to other field invariants and their behavior under quadratic extensions. We recover in particular Prestel's corresponding result on the pythagoras number. Recall that the pythagoras number of $K$, denoted $p(K)$, is defined as the least positive integer $n$ such that any element of $\sum K^{2}$ can be written as a sum of $n$ squares in $K$, with the convention that $p(K)=\infty$ if no such integer exists.

Our construction given in (5.1) below is more explicit and simpler than the one in [12]. We only need to construct a base field (as an extension of a given field) containing a certain element and then derive the conclusions for the quadratic extension from there.

5.1. Theorem. Given a field $k$ and an ordering $P_{0}$ of $k$, there is a field extension $K / k$ of transcendence degree 1 and an element $d \in K^{\times}$such that $K^{2}-d K^{2}$ is an ordering of $K$ extending $P_{0},\left(K^{\times 2} \cup d K^{\times 2}\right) \cap k^{\times}=k^{\times 2}$, and $D_{K}\langle 1, a\rangle D_{K}\langle 1, a d\rangle=$ $\left(K^{\times 2} \cup d K^{\times 2}\right) D_{k}\langle 1, a\rangle$ for every $a \in k^{\times}$.

Proof: $\quad$ Let $\hat{k}=k((t))(\sqrt[n]{t} \mid n \in \mathbb{N} \backslash\{0\})$ and let $v: \hat{k} \longrightarrow \mathbb{Q} \cup\{\infty\}$ be the $k$-valuation with $v(t)=1$. This valuation is henselian. Let $H$ denote the relative algebraic closure of $k(t)$ inside $\hat{k}$. The restricted valuation $\left.v\right|_{H}: H \longrightarrow \mathbb{Q} \cup\{\infty\}$ is henselian by $[7,(4.1 .5)]$, and it also has value group $\mathbb{Q}$ and residue field $k$. It follows easily that $H^{\times}=k^{\times} H^{\times 2}$ and $k^{\times} \cap H^{\times 2}=k^{\times 2}$, and further that every anisotropic quadratic form over $k$ stays anisotropic over $H$. In particular, the inclusion $k \subseteq H$ induces an isomorphism $k^{\times} / k^{\times 2} \longrightarrow H^{\times} / H^{\times 2}$.

There is a unique ordering $P_{0}^{\prime}$ of $k(t)$ such that $P_{0}^{\prime} \cap k=P_{0}$ and $t-a \in P_{0}^{\prime}$ for all $a \in k$. We set $d=1-t$. Since $-d \in P_{0}^{\prime}$ and $d \in H \cap \hat{k}^{2}=H^{2}$, we have $-1 \in P_{0}^{\prime} \cdot H^{2}$. Hence $P_{0}^{\prime}$ does not extend to an ordering of $H$. Using Zorn's Lemma, we choose a maximal extension of ordered fields $(K, P)$ of $\left(k(t), P_{0}^{\prime}\right)$ with $K \subseteq H$. The choice implies that $P^{\times} \cap H^{\times 2}=K^{\times 2}$. Note that $K$ is an algebraic extension of $k(t)$ and therefore has transcendence degree one over $k$.

Let $G=K^{\times} \cap H^{\times 2}$. Then $G \cap P^{\times}=K^{\times 2}$ and the inclusions $k \subseteq K \subseteq H$ induce natural isomorphisms

$$
k^{\times} / k^{\times 2} \longrightarrow K^{\times} / G \longrightarrow H^{\times} / H^{\times 2} .
$$

As $d \in G \backslash P^{\times}$we have $K^{\times}=G P^{\times}$, hence $K^{\times} / P^{\times} \simeq G /\left(G \cap P^{\times}\right)=G / K^{\times 2}$ and thus $\left[G: K^{\times 2}\right]=2$ and $G=K^{\times 2} \cup d K^{\times 2}$. Hence $\left(K^{\times 2} \cup d K^{\times 2}\right) \cap k^{\times}=G \cap k^{\times}=$ $k^{\times 2}$. Moreover, $H^{\times}=k^{\times} H^{\times 2}$ and any anisotropic form over $k$ stays anisotropic over $H$. For any $a \in k^{\times}$we obtain that $D_{H}\langle 1, a\rangle=H^{\times 2} D_{k}\langle 1, a\rangle$ and thus

$$
D_{K}\langle 1, a\rangle D_{K}\langle 1, a d\rangle \subseteq K^{\times} \cap D_{H}\langle 1, a\rangle=\left(K^{\times} \cap H^{\times 2}\right) D_{k}\langle 1, a\rangle=G D_{k}\langle 1, a\rangle,
$$


and as the opposite inclusion is obvious, we have equality.

As $d \in G$ we have $K^{\times}=k^{\times} G=\left(P_{0}^{\times} \cup-P_{0}^{\times}\right) G=\left(P_{0}^{\times} \cup-d P_{0}^{\times}\right) G$. Since $P_{0}^{\times} \cup-d P_{0}^{\times} \subseteq P^{\times}$we obtain that

$$
P^{\times}=\left(\left(P_{0}^{\times} \cup-d P_{0}^{\times}\right) G\right) \cap P^{\times}=\left(P_{0}^{\times} \cup-d P_{0}^{\times}\right)\left(G \cap P^{\times}\right)=\left(P_{0}^{\times} \cup-d P_{0}^{\times}\right) K^{\times 2} .
$$

For $a \in P_{0}^{\times}$we have $-d a\left(t-a^{-1}\right)=d(1-a t) \in P^{\times} \cap H^{\times 2}=K^{\times 2}$, so that $1-$ at $\in d K^{\times 2}$ and thus at $\in K^{2}-d K^{2}$, and since $t \in K^{\times 2}$, we obtain that $a \in K^{2}-d K^{2}$. This shows that $P_{0} K^{2} \subseteq K^{2}-d K^{2}$. It follows that $P=\left(P_{0} \cup-d P_{0}\right) K^{2} \subseteq K^{2}-d K^{2}$. Since $-d \in P$ we thus have $P=K^{2}-d K^{2}$.

5.2. Lemma. Let $K / k$ be a field extension and $G$ a subgroup of $K^{\times}$such that $K^{\times 2} \subseteq G, G k^{\times}=K^{\times}$, and $G+a G=G \cdot D_{k}\langle 1, a\rangle$ for any $a \in k^{\times} \backslash-k^{\times 2}$. Then the following hold:

(a) If $n \in \mathbb{N}$ and $a_{1}, \ldots, a_{n} \in k^{\times}$are such that $\left\langle a_{1}, \ldots, a_{n}\right\rangle$ is anisotropic over $k$, then for any $g_{1}, \ldots, g_{n} \in G$ the form $\left\langle a_{1} g_{1}, \ldots, a_{n} g_{n}\right\rangle$ over $K$ is anisotropic.

(b) If $T$ is preordering of $k$, then $T G$ is the unique preordering $T^{\prime}$ of $K$ with $G \subseteq T^{\prime \times}$ and $T^{\prime} \cap k=T$. In particular, if $k$ is real, then so is $K$.

(c) If $T$ is a fan of $k$, then $T G$ is a fan of $K$ with $\operatorname{deg}(T G)=\operatorname{deg}(T)$.

(d) One has $p(K) \geq p(k)$. Moreover, if $k$ is real and $G \cap \sum K^{2}=K^{\times 2}$, then $p(K)=p(k)$.

Proof: Consider $n \in \mathbb{N}$ and $a_{1}, \ldots, a_{n} \in k^{\times}$such that $\left\langle a_{1}, \ldots, a_{n}\right\rangle$ is anisotropic. We claim that $a_{1} G+\cdots+a_{n} G \subseteq G \cdot D_{k}\left\langle a_{1}, \ldots, a_{n}\right\rangle$. As $0 \notin G \cdot D_{k}\left\langle a_{1}, \ldots, a_{n}\right\rangle$, showing this claim will in particular prove $(a)$. The claim is trivial for $n \leq 1$ and clear from the hypothesis for $n=2$. We proceed by induction on $n$. Suppose that $n \geq 3$. Let $c \in a_{1} G+\cdots+a_{n} G$ be given. As by the induction hypothesis we have $a_{1} G+\cdots+a_{n-1} G \subseteq G \cdot D_{k}\left\langle a_{1}, \ldots, a_{n-1}\right\rangle$, we have that $c=b G+a_{n} G$ for some $b \in D_{k}\left\langle a_{1}, \ldots, a_{n-1}\right\rangle$. Being a subform of $\left\langle a_{1}, \ldots, a_{n}\right\rangle$, the form $\left\langle b, a_{n}\right\rangle$ over $k$ is anisotropic, so that $c \in b G+a_{n} G \subseteq G \cdot D_{k}\left\langle b, a_{n}\right\rangle \subseteq G \cdot D_{k}\left\langle a_{1}, \ldots, a_{n-1}\right\rangle$. This shows the claim and therefore part $(a)$.

Before proving $(b)$ and $(c)$ we need several observations. For $c \in k^{\times} \backslash k^{\times 2}$ the hypothesis yields that $G-c G=G \cdot D_{k}\langle 1,-c\rangle$, and as this set does not contain 0 , we obtain that $c \notin G$. This shows that $k^{\times} \cap G=k^{\times 2}$ and the inclusion $k^{\times} \subseteq K^{\times}$induces a group isomorphism $k^{\times} / k^{\times 2} \longrightarrow K^{\times} / G$. Furthermore, a bijection between the subgroups $H$ of $k^{\times}$containing $k^{\times 2}$ and the subgroups $H^{\prime}$ of $K^{\times}$containing $G$ is given by $H \mapsto G H$, with inverse given by $H^{\prime} \mapsto H^{\prime} \cap k^{\times}$.

To prove part $(b)$, consider a preordering $T$ of $K$. Using the isomorphism $k^{\times} / k^{\times 2} \longrightarrow K^{\times} / G$, we obtain that $S=T G$ is the unique multiplicatively closed subset $S$ of $K$ with $G \subseteq S$ and $S \cap k=T$. In particular $-1 \notin T G$. For any $a \in T^{\times}$ we have $G+a G=G D_{k}\langle 1, a\rangle \subseteq G\left(T^{\times}+T^{\times}\right)=G T^{\times}$, whence $G+T^{\times} G \subseteq G T^{\times}$. Therefore $T^{\times} G+T^{\times} G=T^{\times}\left(G+T^{\times} G\right) \subseteq T^{\times} G$. Hence, $T G$ is a preordering. Finally, if $k$ is real, then $T=\sum k^{2} \cup\{0\}$ is a preordering of $k$, and thus the above argument yields that $K$ is real as well. 
To prove $(c)$, assume now that $T$ is a fan of $k$. By Condition $(i i)$ in (2.1), every multiplicatively closed subset of $k$ containing $T$ and not containing -1 is a preordering of $k$. Consider a multiplicatively closed subset $S$ of $K$ with $T G \subseteq S$ and $-1 \notin S$. Then $T \subseteq S \cap k$, so $S \cap k$ is a preordering of $k$. By part $(b)$, then $S=(S \cap k) G$ is a preordering of $K$. In view of Condition (ii) in (2.1), this argument shows that $T G$ is a fan. The isomorphism $k^{\times} / k^{\times 2} \longrightarrow K^{\times} / G$ now shows that $\operatorname{deg}(T G)=\left[K^{\times}: \pm(T G)^{\times}\right]=\left[k^{\times}: \pm T^{\times}\right]=\operatorname{deg}(T)$.

For $(d)$, as any anisotropic form over $k$ stays anisotropic over $K$ by part $(a)$, we have that $p(K) \geq p(k)$. Assume finally that $k$ is real. Then by part $(c)$, $G \sum k^{2} \cup\{0\}$ is a preordering, thus $\sum K^{2} \subseteq G \sum k^{2}$. From this it follows that $\sum K^{2}=\left(G \cap \sum K^{2}\right) \sum k^{2}$. Hence, if $G \cap \sum \bar{K}^{2}=K^{\times 2}$, then $p(K)=p(k)$.

5.3. Theorem. Let $K / k$ be a field extension and $d \in K^{\times}$such that $K^{2}-d K^{2}$ is an ordering of $K,\left(K^{\times 2} \cup d K^{\times 2}\right) \cap k^{\times}=k^{\times 2}$, and such that $D_{K}\langle 1, a\rangle D_{K}\langle 1, a d\rangle=$ $\left(K^{\times 2} \cup d K^{\times 2}\right) D_{k}\langle 1, a\rangle$ for every $a \in k^{\times}$. Then $K^{\times}=\left(k^{\times} \cup d k^{\times}\right) K^{\times 2}$, and for any two forms $\varphi$ and $\psi$ over $k$ such that $\varphi \perp \psi$ is anisotropic, the form $\varphi_{K} \perp d \psi_{K}$ over $K$ is anisotropic. Furthermore, $L=K(\sqrt{-d})$ is a quadratic extension of $K$ such that the following hold:

(a) $|X(K)|=|X(k)|+1$ and $|X(L)|=2$.

(b) $\operatorname{st}(K)=\max \{s t(k), 1\}$ and $\operatorname{st}(L)=1$.

(c) $\left|K^{\times} / K^{\times 2}\right|=2 \cdot\left|k^{\times} / k^{\times 2}\right|$ and $\left|L^{\times} / L^{\times 2}\right|=2 \cdot\left|k^{\times} / k^{\times 2}\right| \cdot\left|D_{k}(\langle 1,1\rangle) / k^{\times 2}\right|$.

(d) $p(K)=p(k)$, and if $\left|k^{\times} / k^{\times 2}\right|=2$ then $p(L)=1$, otherwise $p(L)=2$.

Proof: Let $G=K^{\times 2} \cup d K^{\times 2}$ and $P=K^{2}-d K^{2}$. For $a=-1$ the hypothesis yields that $K^{\times}=D_{K}\langle 1,-1\rangle=G \cdot D_{k}\langle 1,-1\rangle=G \cdot k^{\times}$. For $a \in k^{\times} \backslash-k^{\times 2}$, the hypotheses yield that $-a \notin G$ and $G+a G \subseteq D_{K}\langle 1, a\rangle D_{K}\langle 1, a d\rangle=G \cdot D_{k}\langle 1, a\rangle \subseteq G+a G$, and therefore $G+a G=G \cdot D_{k}\langle 1, a\rangle$. Hence, the hypotheses of (5.2) are satisfied.

The statements on anisotropy of quadratic forms over $k$ and $K$ follow directly from part (a) of (5.2).

As $d \in G \backslash P^{\times}$we have $K^{\times}=G P^{\times}$, hence $K^{\times} / P^{\times} \simeq G /\left(G \cap P^{\times}\right)=G / K^{\times 2}$ and $\left[G: K^{\times 2}\right]=\left[K^{\times}: P^{\times}\right]=2$. Therefore $\left|K^{\times} / K^{\times 2}\right|=2 \cdot\left|K^{\times} / G\right|=2 \cdot\left|k^{\times} / k^{\times 2}\right|$. Since $G \cap P^{\times}=K^{\times 2}$ we have $G \cap \sum K^{2}=K^{\times 2}$, so that $p(K)=p(k)$ by (5.2). Furthermore, by (5.2) for any fan $T$ of $k$ the set $T G=(T \cup d T) K^{2}$ is a fan of $K$ with $\operatorname{deg}(T G)=\operatorname{deg}(T)$, and conversely any fan of $K$ containing $d$ is obtained in this way. For fans of degree zero this means that every ordering of $k$ extends uniquely to an ordering of $K$ containing $d$. Since $P$ is the only ordering of $K$ containing $-d$, we conclude that $|X(K)|=|X(k)|+1$. In particular, st $(K) \geq 1$. For any fan $T^{\prime}$ of $K$ with $d \notin T^{\prime}$, we have that $T^{\prime}-d T^{\prime}$ is a fan of $K$ containing $K^{2}-d K^{2}=P$, hence $T^{\prime}-d T^{\prime}=P$ and therefore $\left[P^{\times}: T^{\prime \times}\right] \leq 2$, showing that $\operatorname{deg}\left(T^{\prime}\right) \leq 1$. For a fan $T^{\prime}$ of $K$ with $d \in T^{\prime}$, we have that $T=T^{\prime} \cap k$ is a fan of $k$ by $(2.9)$, and as $T^{\prime}=(T \cup d T) K^{2}$ the natural map $k^{\times} / T^{\times} \longrightarrow K^{\times} / T^{\prime \times}$ is an isomorphism, so that $\operatorname{deg}\left(T^{\prime}\right)=\operatorname{deg}(T)$. Since $s t(K) \geq 1$ we conclude that $\operatorname{st}(K)=\max \{s t(k), 1\}$. 
Since $-1 \notin k^{\times 2}=k^{\times} \cap\left(K^{\times 2} \cup d K^{\times 2}\right)$ we have $-d \notin K^{\times 2}$, so $L=K(\sqrt{-d})$ is a quadratic extension of $K$. The only orderings of $L$ are the two extensions of $P$. In particular $|X(L)|=2$ and $s t(L)=1$. As $D_{K}\langle 1,1\rangle \subseteq P^{\times}=D_{K}\langle 1,-d\rangle$ we obtain that $D_{K}\langle 1,1\rangle \subseteq D_{K}\langle 1, d\rangle$, using e.g. [10, Chap. I, Ex. 24]. Since $D_{K}\langle 1, d\rangle \subseteq G+G=G D_{k}\langle 1,1\rangle$, we obtain that $D_{K}\langle 1, d\rangle=G D_{k}\langle 1,1\rangle$. Since $G \cap k^{\times}=k^{\times 2}$ we conclude that

$$
D_{K}\langle 1, d\rangle / G \simeq G D_{k}\langle 1,1\rangle / G \simeq D_{k}\langle 1,1\rangle / k^{\times 2}
$$

and thus $\left|D_{K}\langle 1, d\rangle / K^{\times 2}\right|=2 \cdot\left|D_{k}\langle 1,1\rangle / k^{\times 2}\right|$. By [10, Chap. VII, (3.8)] we obtain that

$$
\left|L^{\times} / L^{\times 2}\right|=\frac{1}{2} \cdot\left|K^{\times} / K^{\times 2}\right| \cdot\left|D_{K}\langle 1, d\rangle / K^{\times 2}\right|=\left|k^{\times} / k^{\times 2}\right| \cdot 2 \cdot\left|D_{k}(\langle 1,1\rangle) / k^{\times 2}\right| .
$$

Since $N_{L / K}\left(\sum L^{2}\right) \subseteq \sum K^{2} \subseteq P^{\times}=D_{K}\langle 1,-d\rangle$ where $N_{L / K}: L \longrightarrow K$ is the norm map, the Norm Principle [10, Chap. VII, (5.10)] shows that

$$
\sum L^{2} \subseteq K^{\times} D_{L}\langle 1,-d\rangle=K^{\times} D_{L}\langle 1,1\rangle .
$$

Since $K^{\times} \cap \sum L^{2} \subseteq P^{\times}=D_{K}\langle 1,-d\rangle \subseteq D_{L}\langle 1,1\rangle$, we obtain that $\sum L^{2}=D_{L}\langle 1,1\rangle$, whence $p(L) \leq 2$. Finally, $p(L)=1$ if and only if $P^{\times}=K^{\times 2} \cup-d K^{\times 2}$, if and only if $\left|K^{\times} / K^{\times 2}\right|=4$, if and only if $\left|k^{\times} / k^{\times 2}\right|=2$.

5.4. Remark. It follows from (5.3) that the construction of (5.1) gives a particularly explicit realization of a Witt product of two Witt rings of fields as the Witt ring of another field. Starting with a real field $k$, in the proof of (5.1) an extension $H / k$ was constructed such that the scalar extension from $k$ to $H$ yields an isomorphism of Witt rings $W k \longrightarrow W H$. Then an intermediate field $K$ and an ordering $P$ of $K$ were found such that the scalar extension from $K$ to $H$ and the signature at $P$ together yield an isomorphism of the Witt ring $W K$ with the Witt product of $W k$ with $\mathbb{Z}$ (which can be interpreted as the Witt ring of the real closure of $K$ at $P$ ). More generally, it was shown in [8] (though in terms of quadratic form schemes) that any Witt ring product of two Witt rings of fields is the Witt ring of another field, but the general construction is more involved and not as explicit.

5.5. Corollary. Let $n, r \in \mathbb{N} \cup\{\infty\}$ with $r>0$. There exists a quadratic field extension $L / K$ such that $K$ is pythagorean, st $(K)=n+r$ and $s t(L)=n+1$, $|X(K)|=2^{n}\left(2^{r}+1\right)$ and $|X(L)|=2^{n+1}$, and $\left|K^{\times} / K^{\times 2}\right|=\left|L^{\times} / L^{\times 2}\right|=2^{n+r+2}$.

Proof: Assume first that $n$ and $r$ are finite. Let $k=\mathbb{R}\left(\left(t_{1}\right)\right) \ldots\left(\left(t_{r}\right)\right)$. By $(2.7) k^{2}$ is a fan of degree $r$, so $k$ is pythagorean with $s t(k)=r$ and $\left|k^{\times} / k^{\times 2}\right|=2^{r+1}$. Let $K_{0} / k$ be a field extension obtained together with an element $d \in K_{0}^{\times}$by using (5.1), and let $L_{0}=K_{0}(\sqrt{d})$. Using the properties of the extension $L_{0} / K_{0}$ that follow by (5.3), it follows by induction on $n$ from (2.6) that $K=K_{0}\left(\left(X_{1}\right)\right) \ldots\left(\left(X_{n}\right)\right)$ and $L=L_{0}\left(\left(X_{1}\right)\right) \ldots\left(\left(X_{n}\right)\right)$ have the desired properties. The same argument works in case $n$ or $r$ are infinite when passing to infinitely iterated power series fields where necessary. 
As noted in $[14,(3.2)]$, alternatively, the theory of spaces of orderings can be used to obtain the part of the statement of (5.5) concerning the stability index.

5.6. Remark. Part of (5.5) also holds for $r=0$, by a different argument. For $n \in \mathbb{N}$ we gave in (2.8) an easy example of a quadratic extension $L / K$ where $\sum K^{2} \cup\{0\}$ is a fan of degree $n$ of $K$ and $\sum L^{2} \cup\{0\}$ is a fan of degree $n+1$ of $L$, thus in particular with $s t(K)=n$ and $s t(L)=n+1$.

5.7. Corollary. The possible pairs of values $(s t(K)$, st $(L))$ for real quadratic extensions $L / K$ are $(n+r, n+1)$ with $n, r \in \mathbb{N} \cup\{\infty\}$.

Proof: For any algebraic extension $L / K$ one has $s t(L) \leq s t(K)+1$ by $[4,(4.3)]$. For a real quadratic extension $L / K$ one has $s t(L) \geq 1$ as $L$ cannot be uniquely ordered. This together with (5.5) and (5.6) shows the statement.

Acknowledgments. This work was supported by the Deutsche Forschungsgemeinschaft (project Quadratic Forms and Invariants, BE 2614/3) and by the Zukunftskolleg, Universität Konstanz.

\section{REFERENCES}

[1] K.J. Becher. Totally positive extensions and weakly isotropic forms. Manuscripta Math. 120 (2006): 83-90.

[2] E. Becker, E. Köpping. Reduzierte quadratische Formen und Semiordnungen reeller Körper. Abh. Math. Sem. Univ. Hamburg 46 (1977): 143-177.

[3] K.J. Becher, D.B. Leep. Real fields, valuations, and quadratic forms. Preprint 2011, http://www .mathematik. uni-bielefeld.de/lag/man/434.html.

[4] L. Bröcker. Zur Theorie der quadratischen Formen über formal reellen Körpern. Math. Ann. 210 (1974): 233-256.

[5] L. Bröcker. Characterization of fans and hereditarily Pythagorean fields. Math. Z. 151 (1976): 149-163.

[6] R. Elman, T.Y. Lam, A.R. Wadsworth. Orderings under field extensions. J. Reine Angew. Math. 306 (1979): 7-27.

[7] A.J. Engler, A. Prestel. Valued fields. Springer Monographs. Springer-Verlag, Berlin, 2005.

[8] M. Kula. Fields and quadratic form schemes. Ann. Math. Sil. 13 (1985): 7-22.

[9] T.Y. Lam. Orderings, valuations and quadratic forms. CBMS Regional Conference Series in Math., 52. Amer. Math. Soc., Providence, RI, 1983.

[10] T.Y. Lam. Introduction to quadratic forms over fields. Graduate Studies in Mathematics, 67, Amer. Math. Soc., Providence, RI, 2005.

[11] A. Prestel. Quadratische Semi-Ordnungen und quadratische Formen. Math. Z. 133 (1973): 319-342.

[12] A. Prestel. Remarks on the Pythagoras and Hasse number of real fields. J. Reine Angew. Math. 303/304 (1978): 284-294.

[13] A. Prestel. Lectures on Formally Real Fields. Lecture Notes in Mathematics, 1093, Springer-Verlag, Berlin, 1984.

[14] C. Schubert. Weakly isotropic quadratic forms under field extensions. Math. Z. 269 (2011): 137-146. 
Universität Konstanz, Zukunftskolleg / FB Mathematik und Statistik, 78457 Konstanz, Germany.

E-mail address: becher@maths.ucd.ie

Department of Mathematics, University of Kentucky, Lexington, KY 405060027, USA.

E-mail address: leep@email.uky.edu

Department of Mathematics, SUNY Cortland, Cortland, NY 13045-0900, USA.

E-mail address: schub@ucla.edu 